\title{
Ultrasound estimation of fetal weight in twins by artificial neural network
}

\author{
Hanieh Mohammadi, Meshkat Nemati, Zohreh Allahmoradi, Hoda Forghani Raissi, \\ Somayeh Saraf Esmaili, Ali Sheikhani
}

Department Biomedical Engineering, Science and Research Branch, Islamic Azad University, Tehran, Iran.

Email: H.mohammadi@srbiau.ac.ir

Received 8 November 2010; revised 12 November 2010; accepted 26 November 2010.

\begin{abstract}
This study was undertaken to determine the accuracy of using Ultrasound (US) estimation of twin fetuses by use of Artificial Neural Network. At First, as the training group, we performed US examinations on 186 healthy singleton fetuses within 3 days of delivery. Three input variables were used to construct the ANN model: abdominal circumference (AC), abdominal diameter (AD), biparietal diameter (BPD). Then, a total of 121 twin fetuses were assessed subsequently as the validation group. In validation group, the mean absolute error and the mean absolute percent error between estimated fetal weight and actual fetal weight was $261.77 \mathrm{~g}$ and $7.81 \%$, respectively. Results show that, twin estimation of birth weight by ultrasound correlates fairly well with the actual weights of twin fetuses.
\end{abstract}

Keywords: Ultrasound; Fetal Weight Estimation; Twin; Artificial Neural Network

\section{INTRODUCTION}

Next to preterm labor, intrauterine growth retardation is the most common risk factor leading to perinatal death in twins [1-3]. Estimation of fetal weight by ultrasound is an important investigation in the management of twin pregnancies because of the high incidence of growth retardation [4], the inaccurate estimation of fetal weight by abdominal palpation, and the common excess of amniotic fluid [5]. Growth retardation occurs more frequently in twin gestation than in singletons [6]. This fact contributes heavily to the high frequency of perinatal complications in twin gestation [7]. Weight discrepancy in particular has been shown to be a most significant factor in predicting perinatal mortality and morbidity [8]. Prediction of fetal weight by Ultrasonographic measurements in twin gestation may be complicated by fetal crowding, fetal position and oligohydramnios. Several investigators have commented on the difficulty in obtaining reliable measurements of the fetal biparietal diameter (BPD), and the abdominal circumference (AC) in twins [3-5]. The most widely used weight formula for both singletons and twins is the Shepard et al. revision of the Warsoff et al. equation using the biparietal diameter (BPD) and the abdominal circumference (AC) [1-9]. A slight imprvement of the method has been introduced by incorporating femur length and/or measurements of the head and abdominal areas $[7,8]$. It can, however, be difficult to define the whole AC in twins, and thus diameter measurements may result in more accurate fetal weight estimations [10]. Various neural network architectures and learning methodologies have been used in the literature for fetal weight estimation $[8,10]$. Although different methods are available, a simple, quick and reliable method of assess in birth weight is still under debate [10-13].

Artificial neural network (ANN), a computerized analog of a biologic neural system, has been widely used in many different professional fields [5-7]. The constructed architecture of the ANN model would develop relationship between the input and output data when training proceeds. The way of training the ANN model simulates how biologic neural connections are established and rectified perpetually. After an appropriate training process, the nonlinear neural network can afford a best fit guess as a result. The architecture, principles, characteristics and applications have been discussed in the literature [10-26]. The purpose of this study was to determine the accuracy of using ANN model in predicting fetal weight in Twins and probably develop a new way which may be more relevant to assess accurate fetal weight estimation and could develop nonlinear relationships between input variables and output outcomes and reduce the errors between estimated fetal weight and actual fetal weight. In this paper we try to evaluate the accuracy of 
using ANN model together with BPD, AC, and AD as inputs for developing nonlinear relationships between input variables and output outcomes and reduce the errors between estimated fetal weight and actual fetal weight in twins.

\section{MATERIALS AND METHODS}

A retrospective study of 186 consecutive twin pregnancies delivered in the department of Obstetrics and Gynecology at Madaran medical faculty hospital, between January 2010 and April 2010, was undertaken. In all cases the estimated date of confinement had been established by ultrasound scan at 20 weeks of gestation. Median maternal age was 26.7 years (range 15-44), median number of pregnancies was 2 (1-5), number of previous deliveries $0(0-3)$, and median gestational age at delivery was 36 weeks (14-41). The median birth weight of twin A was $2390 \mathrm{~g}$ (160-2918 g), and of twin B $2265 \mathrm{~g}$ (210-2868 g). We consecutively performed fetal biometry by US on every healthy twin fetuses which was admitted to the delivery room. The exclusion criteria were fetal anomaly and fetuses not delivered within 3 days of US examination [2]. All women had a normal pregnancy with ultrasound documentation of the BPD, AC and AD $[8,10,14]$. A total of 189 fetuses that met the above criteria were included as the training group of the MNM model. For further validate the established ANN model in fetal weight estimation 81 fetuses that were delivered within the subsequent 3 months from May 2010 to July 2010 and met the criteria described above were used as the validation group. The fetal BPD was measured according to Watmough et al. [10], from the outer to the inner contour of the head. The fetal AD was calculated as a mean of two diameters at right angles to one another, measured on the outer contour on a transverse scan of the fetal long axis, and at the level where the umbilical vein enters the ductus venosus $[2,11]$. Also, the fetal abdominal circumference (AC) was measured at the level of the umbilical vein entry into the ductus venosus [2]. The measurements were done 4 times and rounded to the nearest millimeter. All the US measurements were conducted by experienced sonographist according to the methods previously described. We used the commercially available 2-D US scanners (Aloka SSD-650) with a $3.5-\mathrm{MHz}$ transabdominal probe.

\section{ANN MODEL}

A neural network is a model that simulates the functions of biologic neurons. The ability of a single neuron could be greatly improved by connecting multiple neurons in a layer. Artificial neural networks are powerful non-linear models used vastly for classifying different types of data. A neural network is composed of few layers of neurons.
Neurons in adjusting layers are connected with relative quantitative weights. These weights are randomly chosen, and they are changed through the training procedure, so that the mean of the sum-of-squares error (MSE) is minimized. The MSE is the squared difference between the network output and network target, averaged over all of the cases [16,17]. Figure 1 shows the architecture of the trained ANN model and Figure 2 is the neural network development flow chart. Our trained ANN model in our investigation was composed of four layers: 1 ) one input layer with three inputs; 2) hidden layer with 6 neurons and 18 connections; 3 ) hidden layer with 2 neurons and 12 connections 3 . One output layer with one outcome and 2 connections. The back propagation (BPN) network algorithm is used as the learning algorithm to train the artificial neural network $[9,10]$.

Three inputs were BPD, AC, and AD. Furthermore, to examine the performance of ANN model we calculate mean absolute error (AE) and mean absolute percent error (APE) between actual fetal weight and estimated fetal weight.

\section{RESULT}

The median birth weight of twin A was $2390 \mathrm{~g}$ (150$3200 \mathrm{~g})$, and of twin B $2165 \mathrm{~g}$ (210-3250 g) in the validation group, respectively. In validation group, the mean absolute error (AE) and the mean absolute percent error (APE) between estimated fetal weight and actual fetal weight was $261.77 \mathrm{~g}$ and $7.81 \%$. Also, the moderation significant correlations between the actual fetal weight and the estimated fetal weight of the validation group are shown in Figure $3(r=0.9348, n=121)$. Also, the overall, high correlation between AC, AD, BPD and twin`s EFW were $0.81,0.87$ and 0.84 , respectively, which shows the important effect of these parameters on twin`s weight. It seems that the prediction error is known to increase with increasing twin's weight.

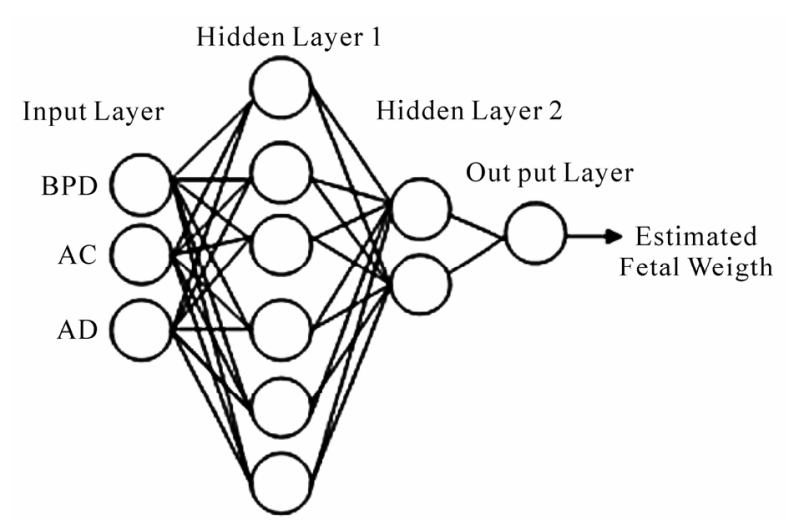

Figure 1. Architecture of the trained ANN model, BPD = biparietal distance, $\mathrm{AC}=$ abdominal circumference, $\mathrm{AD}=$ abdominal diameter. 


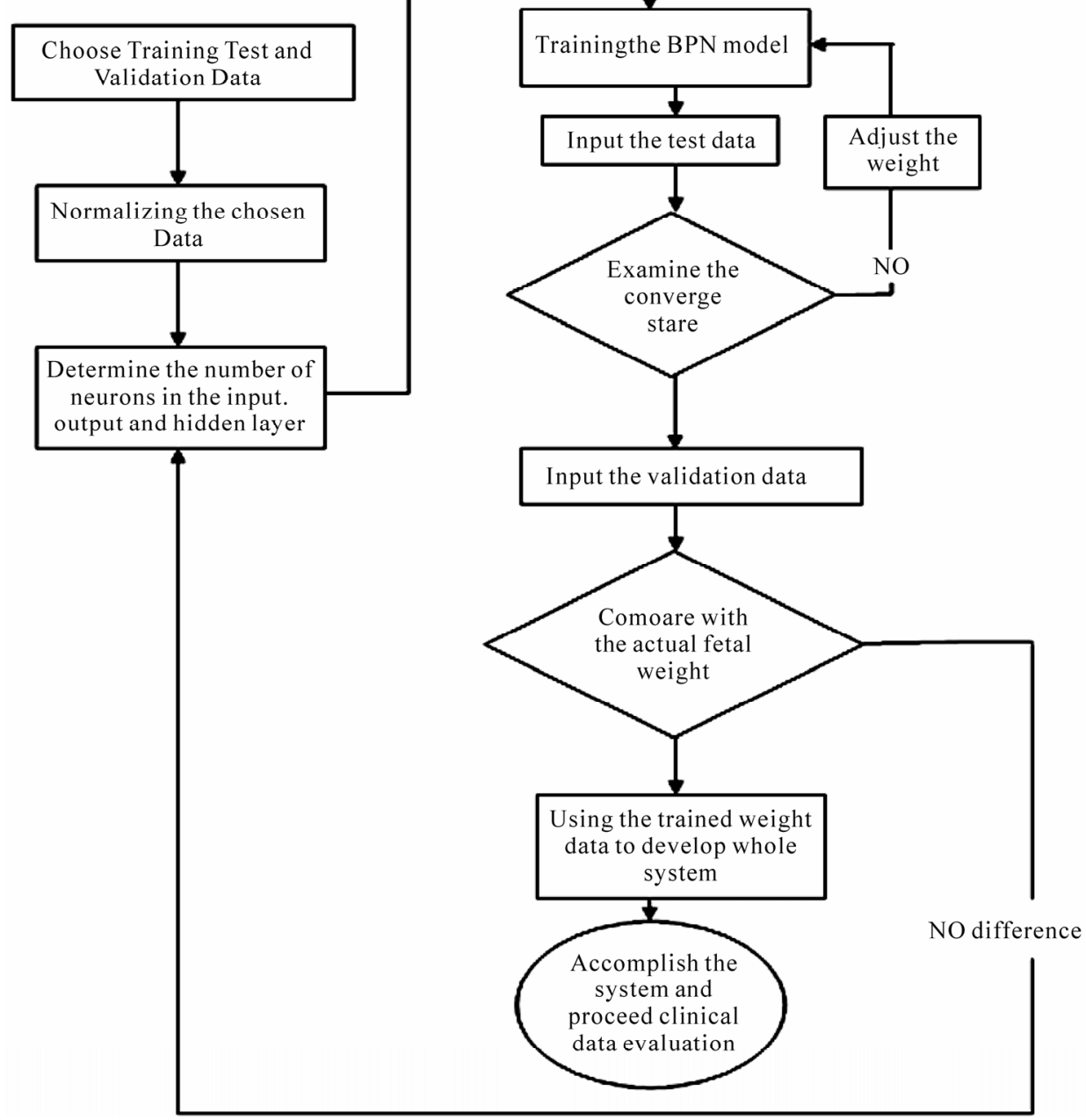

Figure 2. The neural network development flow chart.

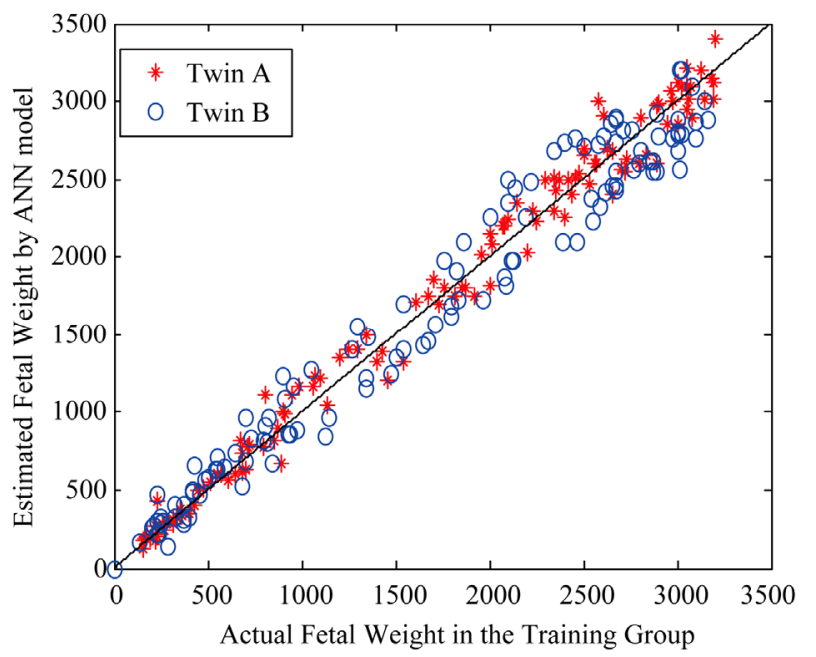

Figure 3. Scattergram of the estimated fetal body weight vs. the actual fetal body weight the by the ANN model in the validation group.

\section{CONCLUSIONS}

A neural network is a model that simulates the functions of biologic neurons. The ability of a single neuron could be greatly improved by connecting multiple neurons in layers. Artificial neural networks are powerful non-linear models used vastly for classifying different types of data. A neural network is composed of few layers of neurons. Neurons in adjusting layers are connected with relative quantitative weights. These weights are randomly chosen, and then are changed through the training procedure, so that the mean of the sum-of-squares error (MSE) is minimized [2,11,12]. The ANNs learning algorithms can be divided into two main groups that are supervised (or Associative learning) and unsupervised (Self-Organization) learning $[2,9,11,14]$. One of the most commonly used supervised ANN model is back propagation (BPN) network that uses back propagation learning algorithm [911]. Back propagation algorithm is one of the well-known 
algorithms in neural networks. The back propagation neural network is essentially a network of simple processing elements working together to produce a complex output [11]. These elements or nodes are arranged into different layers: input, middle and output. The output from a back propagation neural network is computed using a procedure known as the forward pass [2,8-11,15]: 1) The input layer propagates a particular input vector's components to each node in the middle layer. 2) Middle layer nodes compute output values, which become inputs to the nodes of the output layer. 3) The output layer nodes compute the network output for the particular input vector. The forward pass produces an output vector for a given input vector based on the current state of the network weights. Since the network weights are initialized to random values, it is unlikely that reasonable outputs will result before training. The weights are adjusted to reduce the error by propagating the output error backward through the network $[2,9,15]$. In this study, we used the BPN algorithm to develop the ANN and prove our hypothesis that BPD, AC and AD within ANN model could reduce errors between estimated fetal weight and actual fetal weight. The subjects in our series were a group of women with healthy singleton fetus with documentation of US examination with, BPD, AC and AD. Some may wonder at our choice of the three input parameters, thinking that they are not well justified. The three dimensional variables are reasonable because of the previous literature [9]. Also, the overall, high correlation between AC, AD, BPD and twin's EFW were 0.81, 0.87 and 0.84 , respectively, which shows the important effect of these parameters on twin's weight. In our study, the definition of an anomaly was for any fetus with a major structural anomaly that could be diagnosed prenatally, such as holoprosencephaly, omphalocele, cystic hygroma, etc. These were excluded from the study. We might include some fetuses with rare and nonstructural anomalies that could only be diagnosed postnatally by genetic screening or metabolic methods, in which the prenatal ultrasonic examination cannot demonstrate any structural abnormality. However, we believe that this point makes only little impact on the study because these nonstructural anomalies are too rare [9].

In our study, the mean absolute error (AE) and the mean absolute percent error (APE) between estimated fetal weight and actual fetal weight were $162.71 \mathrm{~g}$ and $7.81 \%$, respectively. The fetuses in weight range of $(>2500 \mathrm{~g})$ are the lowest accurate fetal weight estimation in validation group $(\mathrm{AE}=269 \mathrm{~g}, \mathrm{APE}=10.51 \%)$, we think that, as the fetus grows are more quick at the last trimester and we considered babies within 3 days of delivery, it might be one part of the error in this weight range is related to fetus grows within this estimation of fetal weight. In this ANN model we have 4 layers; input layer, two median layers and output layer. We have three input variables $\mathrm{AC}, \mathrm{AD}$ and $\mathrm{BPD}$. In all cases the estimated date of confinement had been established by ultrasound scan at 20 weeks of gestation. Median maternal age was 26.7 years (range 15-44), median number of pregnancies was 2 (1-5), number of previous deliveries 0 (0-3), and median gestational age at delivery was 36 weeks (14-41). The median birth weight of twin A was $2390 \mathrm{~g}$ (160-2918 g), and of twin B $2265 \mathrm{~g}$ (210-2868 g) in the training group. Also, the median birth weight of twin A was $2190 \mathrm{~g}$ (150-3200 g), and of twin B $2165 \mathrm{~g}$ (210-2868 g) in the validation group.

Also estimation of fetal weight by ANN model at the weight range of $(<1500 \mathrm{~g})$ are the most accurate result. It seems that the prediction birth weight error is known to increase with increasing weight of twins, week by week. In conclusion, our study demonstrates that our single multiplicative neuron model is a well-established model and can be used to estimate fetal weight. However, more accuracy of fetal weight estimation is in need of further studies.

\section{ACKNOWLEDGEMENTS}

This study was supported in part by grants from university of science and research branch Islamic Azad University, Tehran. The authors are grateful to all doctors for the ultrasound measurements; Ms. Fatemeh Nematollahi and Ms. Fatemeh Bani and their assistance; and Ali Ghafari at the Department of Obstetrics and gynecology, Madaran Medical Faculty for equipment supply.

\section{REFERENCES}

[1] Hendricks, C.H. (1966) Twinning in relation to birth weight, mortality and congenital anomalies. Obstetrics Gynecology, 27, 47-53.

[2] Powers, W.F. (1973) Twin pregnancy, complications and treatment. Obstetrics Gynecology, 43, 795-808.

[3] Chang, F.M., Liang, R.I., Ko, H.C., Yao, B.L., Chang, C.H. and Yu, C.H. (1997) Threedimensional ultrasound- assessed fetal thigh volumetry in predictingbirth weight, $\mathrm{Ob}$ stetrics Gynecology, 90, 331-339. doi:10.1016/S0029-7844(97)00280-9

[4] Manlan, G. and Scott, K.E. (1978) Contribution of twin pregnancy to perinatal mortality and fetal growth, retardation: reversal of growth retardation after birth. Canadian Medical Association Journal, 118, 365-368.

[5] Secher, N.J., Kaern, J., Hansen, P.K., et al. (1985) Intra-uterine growth in twin pregnancies: prediction of fetal growth retardation. Obstetrics Gynecology, 66, 63-68.

[6] Shepard, M.J., Richards, V.A., Berkowitz, R.L. Warsof, S.L. and Hobbins, J.C. (1982) An evaluation of two equations for predicting fetal weight by ultrasound. American Journal of Obstetrics Gynecology, 142, 47-54.

[7] Vintzileos, A.M., Campbell, W.A., Rodis, J.F., BorsKoefoed, R. and Nochimson, D.J. (1987) Fetal weight estimation formulas with head, abdominal, femur, and 
thigh circumference measurements. American Journal of Obstetrics Gynecology, 157, 410-414.

[8] Campbell, S. and Wilkin, D. (1975) Ultrasonic measurement of fetal abdomen circumference in the estimation of fetal weight. British Journal of Obstetrics Gynecology, 82, 689-697. doi:10.1111/j.1471-0528.1975.tb00708.x

[9] Secher, N.J., Djursing, H., Kern Hansen, P., et al. (1987) Estimation of fetal weight in the third trimester by ultrasound. European Journal of Obstetrics, Gynecology and Reproductive Biology, 24, 1-11. doi:10.1016/0028-2243(87)90031-1

[10] Sindberg Eriksen, P., Secher, N.J., Weis-Bentzon, M., et al. (1985) Normal growth of the fetal biparietal diameter and the abdominal diameter in a longitudinal study: An evaluation of the two parameters in predicting fetal weight. Acta Obstetricia et Gynecological Scandinavica, 64, 65-70. doi:10.3109/00016348509154690

[11] Jouannic, J.M., Grange, G., Goffinet, F., Benachi, A. and Carbrol, D. (2001) Validity of sonographic formulas for estimating fetal weight below 1, 250g: aseries of 119 cases. Fetal Diagnosis Therapy, 16, 254-258. doi:10.1159/000053923

[12] Hendricks, C.H. (1966) Twinning in relation to birth weight, mortality and congenital anomalies. Obstetrics Gynecology, 7, 47-53.

[13] Eik-Nes, S.H., Groettum, P. and Anderson, N.J. (1982) Estimation of fetal weight by ultrasound measurement. Acta Obstetricia et Gynecological Scandinavica, 61, 307-312.

[14] Bjerkedal, T. and Skjaerven, R. (1980) Percentiles of birth weight and crown-heel length in relation to gestation period of single livebirths. Tidsskrift for den Norske Laegeforening, 100, 88-91.

[15] Chuang, L., Hwang, J.Y., Chang, C.H., Yu, C.H. and Chang, F.M. (2002) U-utrasound estimation of fetal weight with the use of computerized artificial neural network model. Ultrasound in Medicine Biology, 28, 991-996. doi:10.1016/S0301-5629(02)00554-9

[16] Merz, E., Lieser, H., Schicketanz, K.H. and Harle, J. (1988) Intrauterine fetal weight assessment using ultrasound: A comparison of several weight assessment methods and development of a new formula for the determination of fetal weight. Ultraschall in der Medizin, 9, 15-24. doi:10.1055/s-2007-1011588
[17] Schild, R.L., Fell, K., Fimmers, R, Gembruch, U. and Hansmann, M. (1988) A new formula for calculating weight in the fetus of $<$ or $=1600 \mathrm{~g}$. Ultrasound Obstetrics Gynecology, 24, 775-780. doi:10.1002/uog.1741

[18] Farmer, R.M., Medearis, A.L., Hirata, G.I. and Platt, L.D. (1992) The use of a neural net work for the ultrasound estimation of fetal weight in the macrosomic fetus. American Journal of Obstetrics Gynecology, 166, 4671472.

[19] Secher, N.J., Toettrup, A., Djursing, H. and Weber, T. (1989) Fetal weight estimation in twin pregnancies. European Journal of Obstetrics, Gynecology and Reproductive Biology, 32, 181-186. doi:10.1016/0028-2243(89)90033-6

[20] Eriksen, P.S., Secher, N.J. and Weis-Bentzon, M. (1985) Normal growth of the fetal biparietal diameter and the abdominal diameter in a longitudinal study. Acta Obstetricia et Gynecological Scandinavica, 64, 65-70. doi:10.3109/00016348509154690

[21] Halaska, M.G., Vlk, R., Feldmar, P., et al. (2006) Predicting term birth weight using ultrasound and maternal characteristics. European Journal of Obstetrics, Gynecology and Reproductive Biology, 128, 231-235. doi:10.1016/j.ejogrb.2006.01.020

[22] Mielke, G., Pietsch-Breitfeld, B., Salinas, R., Risse, T. and Marzusch, K. (1995) A new formula for prenatal ultrasonographic weight estimation inextreme lypreterm fetuses. Gynecological Obstetric Investigation, 40, 84-88. doi:10.1159/000292311

[23] Yadav, R.N., Kalra, P.K. and John, J. (2007) Time series prediction with single multiplicative neuron model. Applied Soft Computing, 7, 1157-1163. doi:10.1016/j.asoc. 2006.01.003

[24] Houlton, M.C.C., Marivate, M. and Philpott, R.H. (1981) The prediction of fetal growth retardation in twin pregnancy. British Journal of Obstetrics Gynecology, 88, 264-273. doi:10.1111/j.1471-0528.1981.tb00980.x

[25] Blickstein, I. and Lancet, M. (1988) The growth discordant twin. Obstet Gynecol Surv, 43, 509-515. doi:10.1097/00006254-198809000-00002

[26] Pielet, B.W., Sabbagba, E., Scott, N., et al. (1987) Ultrasonic prediction of birth weight in preterm fetuses: Which formula is best? American Journal of Obstetrics Gynecology, 157, 1411-1414. 